This item was submitted to Loughborough's Research Repository by the author.

Items in Figshare are protected by copyright, with all rights reserved, unless otherwise indicated.

\title{
Computed tomography characterisation of additive manufacturing materials
}

PLEASE CITE THE PUBLISHED VERSION

http://dx.doi.org/10.1016/j.medengphy.2010.12.015

\section{PUBLISHER}

(c) IPEM. Published by Elsevier Ltd.

\section{VERSION}

AM (Accepted Manuscript)

\section{LICENCE}

CC BY-NC-ND 4.0

\section{REPOSITORY RECORD}

Bibb, Richard J., Darren Thompson, and John Winder. 2019. "Computed Tomography Characterisation of Additive Manufacturing Materials”. figshare. https://hdl.handle.net/2134/11947. 
This item was submitted to Loughborough's Institutional Repository (https://dspace.lboro.ac.uk/) by the author and is made available under the following Creative Commons Licence conditions.

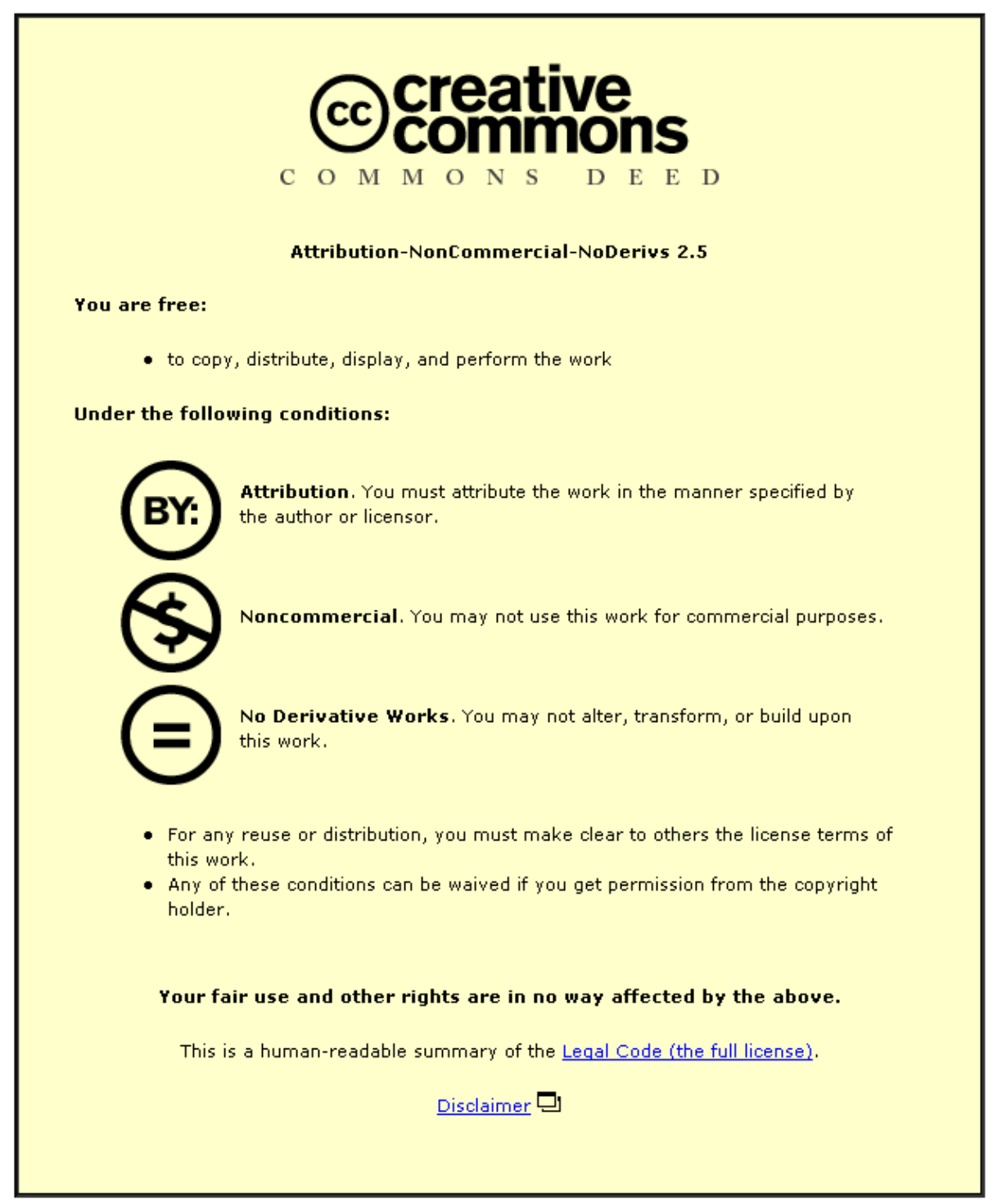

For the full text of this licence, please go to: http://creativecommons.org/licenses/by-nc-nd/2.5/ 
Computed Tomography Characterisation of Additive Manufacturing Materials

\section{Authors}

Richard Bibb BSc PhD AIMPT

Department of Design \& Technology

Loughborough University

Loughborough

Leicestershire

LE11 3TU

United Kingdom

Darren Thompson BSc MSc

Computer Science Department

University of Ulster

Coleraine

BT52 1SA

Northern Ireland

United Kingdom

John Winder BSc MSc PhD PgCHEP CSci MIPEM

Health \& Rehabilitation Sciences Research Institute

School of Health Sciences

University Of Ulster

Shore Road

Newtownabbey

BT37 0QB

United Kingdom 


\section{$\underline{\text { Abstract }}$}

Additive manufacturing, covering processes frequently referred to as rapid prototyping and rapid manufacturing, provides new opportunities in the manufacture of highly complex and custom-fitting medical devices and products. Whilst many medical applications of AM have been explored and physical properties of the resulting parts have been studied, the characterisation of AM materials in computed tomography has not been explored. The aim of this study was to determine the CT number of commonly used AM materials. There are many potential applications of the information resulting from this study in the design and manufacture of wearable medical devices, implants, prostheses and medical imaging test phantoms. A selection of 19 AM material samples were CT scanned and the resultant images analysed to ascertain the materials' CT number and appearance in the images. It was found that some AM materials have CT numbers very similar to human tissues, FDM, SLA and SLS produce samples that appear uniform on CT images and that 3D printed materials show a variation in internal structure. 


\section{Introduction}

Additive Manufacturing (AM) is increasingly used to refer to a variety of technologies that are used to manufacture physical models, prototypes or functional components directly from three-dimensional Computer-Aided Design (3D CAD) data. In AM, physical objects are constructed in a layer-by-layer manner. AM covers all applications and encompasses previous commonly used terms including Rapid Prototyping (RP) and Rapid Manufacturing (RM). It is not appropriate to describe each available AM process here. Full descriptions and technical details are available from the respective manufacturers (a list of contact details and websites is provided below) and there are several texts that provide comprehensive overviews of the processes ${ }^{1,2,3,4}$. Continuous development means that AM technologies can now produce objects in a wide variety of materials ranging from soft, flexible polymers to high performance metal alloys.

AM technologies have been successfully applied in medicine since the early $1990 \mathrm{~s}^{5}$. Initially, RP processes, such as stereolithography, were used to make highly accurate models of skeletal anatomy directly from three-dimensional Computed Tomography (CT) data. Typically referred to as medical modelling or biomodelling, this has now become widely accepted as good practice with many papers published reporting cases and the benefits achieved particularly in cranio-maxillofacial surgery. Medical models have been typically used to plan and rehearse surgery and in the design and manufacture of custom-fitting prostheses. The use of medical models has become commonplace and it is not necessary to discuss these applications in detail here. A number of texts and review papers are available that describe a wide range of medical applications and their principal advantages ${ }^{6,7,8,9,10,11}$.

More recently, AM technologies have been used to directly manufacture custom-fitting medical devices; for example facial prosthetics, removable partial denture frameworks, surgical guides and even implants directly from 3D CAD data ${ }^{12,13,14}$. AM principles are also being exploited in tissue engineering where the advantages of layer additive manufacture are being utilised to build highly complex porous scaffolds that can support the growth of living cells $^{15,16}$. To date no polymer-based AM materials have been specifically developed or approved for implantation and most of the materials tested in this research are not considered biocompatible. The limitations of currently available materials means that this research will be used to provide an indicator of material types that may be further developed for medical applications in the future.

Some assessment of the physical properties of AM materials has been carried out. For example, dimensional accuracy, roughness of surface and mechanical properties has been established for ZPrinter 310 Plus and the Objet Eden 330 ${ }^{17}$. Also, much research has been conducted on the utilisation of CT data in building objects/models using AM technologies. However, the CT properties of AM materials have not been investigated. The characteristics of AM materials under radiological conditions will become important in the future as a variety of medical devices and custom-fitting patient products may be manufactured using $\mathrm{AM}$ and subsequently scanned using CT for either design, testing or treatment purposes. Therefore, the aim of this work was to determine the CT number, (also known as the Hounsfield Unit), of a selection of available AM materials and establish their appearance in CT images. A CT scanner measures the spatial distribution of the linear attenuation coefficient or amount of absorption of X-rays. To enable this measure to be compared between scanners the CT number range was developed which is based on the linear attenuation to X- 
rays of water. The CT number range is typically from -1024 to 3092 (10-bit storage required $)^{18}$. Table 1 shows typical CT numbers from some common human tissues ${ }^{18}$.

\begin{tabular}{|l|c|}
\hline Tissue & CT Number \\
\hline Air & -1005 to -995 \\
\hline Lungs & -950 to -550 \\
\hline Fat & -100 to -80 \\
\hline Water & -4 to 4 \\
\hline Kidney & 20 to 40 \\
\hline Pancreas & 30 to 50 \\
\hline Blood & 50 to 60 \\
\hline Liver & 50 to 70 \\
\hline Spongious Bone & 50 to 300 \\
\hline Compact Bone & $300+$ \\
\hline
\end{tabular}

Table 1: CT numbers of selected human tissues (adapted from Kalender 2000, p.30 Fig 1.9)

\section{$\underline{\text { 2. Materials and Methods }}$}

\subsection{Materials}

A total of 19 AM materials were constructed from a CAD generated STL file defining a rectangular block of material with dimensions $40 \mathrm{~mm}$ x $20 \mathrm{~mm}$ x $10 \mathrm{~mm}$. The samples represented a variety of commonly used materials from the most popular AM processes. However, the sample set is not intended to be comprehensive as there are potentially hundreds of process and material combinations that could have been used. The STL data was sent to a range of AM service providers across the United Kingdom for manufacture (see acknowledgements section for details). Table 2 shows the AM machine, material and physical description of the 19 sample blocks. Fourteen of the blocks were solid and five "sparse" or quasi-hollow. In industry, quasi-hollow parts are typically built to reduce material consumption, build time and therefore cost in some AM processes. In this study for each quasi-hollow sample, there was an equivalent solid sample. The solid samples were used to ascertain CT number ranges and the quasi-hollow samples were included only to investigate their appearance in CT images.

The density of each sample (excluding the "sparse" samples) was calculated by measuring the sample weight in grammes using a Sartorius precision balance and the sample volume in cubic centimetres using a digital Vernier Calliper $\left(\mathrm{g} / \mathrm{cm}^{3}\right)$. 


\begin{tabular}{|c|c|c|c|}
\hline No. & AM Machine & AM Material & Physical Description \\
\hline 1 & Z Corp 450 & Z Bond (cyano-acrylate) & White/grey, opaque, solid \\
\hline 2 & Z Corp 450 & ZP130 (wax) & White/grey, opaque, solid \\
\hline 3 & EOS P100 Formiga & Nylon 12 (polyamide) & White, opaque, solid \\
\hline 4 & 3D Systems 250 solid state laser & ProtoCast AF19120, DSM Somos & Orange/red, translucent, solid \\
\hline 5 & 3D Systems 250 solid state laser & Watershed XC11122, DSM Somos & Clear/blue, translucent, solid \\
\hline 6 & 3D Systems 250 solid state laser & 9420 EP (white), DSM Somos & White/cream, opaque, solid \\
\hline 7 & 3D Systems 250 solid state laser & RenShape SL Y-C 9300, Huntsman & Pink, translucent, solid \\
\hline 8 & 3D Systems InVision SD & VisiJet SR & Clear, translucent, solid \\
\hline 9 & Dimension 1200 SST & ABS & White, opaque, solid \\
\hline 10 & Fortus 400mc & ABS (solid) & White, opaque, solid \\
\hline 11 & Fortus $400 \mathrm{mc}$ & ABS (sparse) & White, opaque, quasi-hollow \\
\hline 12 & Fortus $400 \mathrm{mc}$ & ABS+ (solid) & White, opaque, solid \\
\hline 13 & Fortus $400 \mathrm{mc}$ & ABS+ (sparse) & White, opaque, quasi-hollow \\
\hline 14 & Fortus $400 \mathrm{mc}$ & PPSF (solid) & Light brown, opaque, solid \\
\hline 15 & Fortus $400 \mathrm{mc}$ & PPSF (sparse) & Light brown, opaque, quasi-hollow \\
\hline 16 & Fortus $400 \mathrm{mc}$ & PC (solid) & White, opaque, solid \\
\hline 17 & Fortus $400 \mathrm{mc}$ & PC (sparse) & White, opaque, quasi-hollow \\
\hline 18 & Fortus $400 \mathrm{mc}$ & PC/ABS (solid) & Black, opaque, solid \\
\hline 19 & Fortus $400 \mathrm{mc}$ & PC/ABS (sparse) & Black, opaque, quasi-hollow \\
\hline
\end{tabular}

Table 2: Description of AM material samples

\subsection{CT Scanning}

The AM blocks underwent two Computed Tomography scans. Firstly, they were scanned suspended in air. This was facilitated by using a low-density expanded polystyrene foam support (CT number $=-963)$, as shown in Figure 1 . This low-density foam material has a CT number very similar to air (CT air $=-1000)$ and was selected to minimise any beam hardening effects of the support ${ }^{18,19,20}$. Secondly, the blocks were scanned in contact with a tissue-equivalent head phantom, as shown in Figure 2 (phantom supplied by Imaging Equipment Ltd., Bristol, United Kingdom) to determine any shift to the absolute CT numbers of the samples compared to those measured when scanned in air.

CT scanning was performed using a Philips Brilliance 10 multislice system (www.medical.philips.com) using a sinus/facial/head CT protocol (exposure of 67mAs, peak voltage $120 \mathrm{kV}$, slice thickness $2 \mathrm{~mm}$, rotation time 1 second and convolution kernel type “D”), software version 1.2.0. Whilst a small field of view would have resulted in a smaller pixel size and therefore more pixels within the sample images to analyse, a field of view of $27.9 \mathrm{~cm}$ was specified to replicate the typical field of view, and therefore pixel size, encountered in a wide range of clinical CT applications. CT images were stored in DICOM format and imported into image analysis software (AnalyzeAVW V9.0, Lenexa, Kansas, USA) for CT number measurement. Visual inspection and analysis of the images was also 
performed using another software package (Mimics version 13, Materialise NV, Leuven, Belgium).

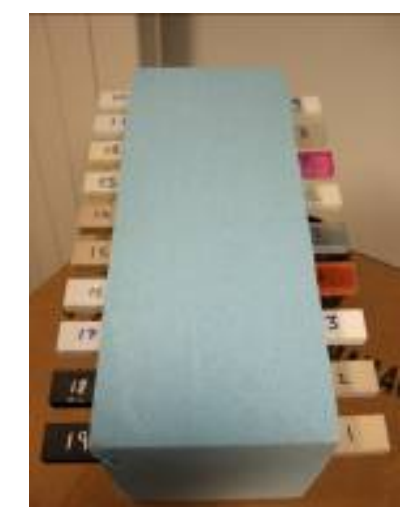

Figure 1: Samples suspended in low-density foam

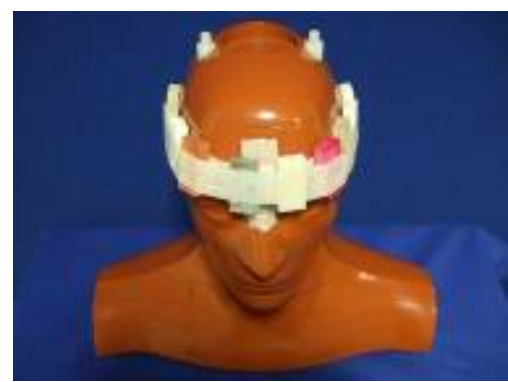

Figure 2: Samples attached to head phantom

\subsection{Data Analysis}

The mean and standard deviation (SD) of the CT numbers for each sample were recorded and averaged over the volume of the material to ensure it was representative of the whole sample. The mean CT numbers measured from the samples scanned in air and positioned on the phantom were compared to determine any shift in CT number due to the presence of the tissue-equivalent phantom. Pixels at the edges of the sample images were not used in the analysis to avoid the partial volume effect, which may lower the average CT number as indicated in Figure 3.



Figure 3: Indicating the image areas analysed and avoided in determining the CT Number

CT images of the sample cross sections were visually inspected to ascertain material structure and any effects of cross sectional variation. Cross sections were visually inspected to determine whether expected material densities were present noting any unexpected features such as voids, porosity or cracking. CT number profiles were also generated to illustrate variation in density. 


\section{$\underline{\text { 3. Results }}$}

Figure 4 shows the relationship between sample density and CT number. The pseudo-hollow sparse samples would obviously have an average sample density much lower than the actual material density, due to the presence of air, and therefore were not included. The result presents the average density for each sample and it should be noted that some samples are not homogeneous and their density varies considerably across their sections (especially the $\mathrm{Z}$ Corp samples). The differences in CT numbers reflect the differences in sample densities. As might be expected, the relationship between CT number and average sample density is essentially linear. It is well known that the CT number of a material is dependent on a range of properties including density, X-ray beam energy and sample thickness. As X-Ray beam energy and section thickness were constant the variations in CT number can be attributed to the differences in material and is related to their density. It can be seen that there is a cluster of samples around the density of 1.0 to $1.2 \mathrm{~g} / \mathrm{cm}^{3}$, which is typical for polymers, and the CT numbers are clustered suggesting that the CT number for these polymers is also similar. The two denser materials are from the $\mathrm{Z}$ Corp process and are not polymers but it is interesting to note that their CT number is also proportional to their density.

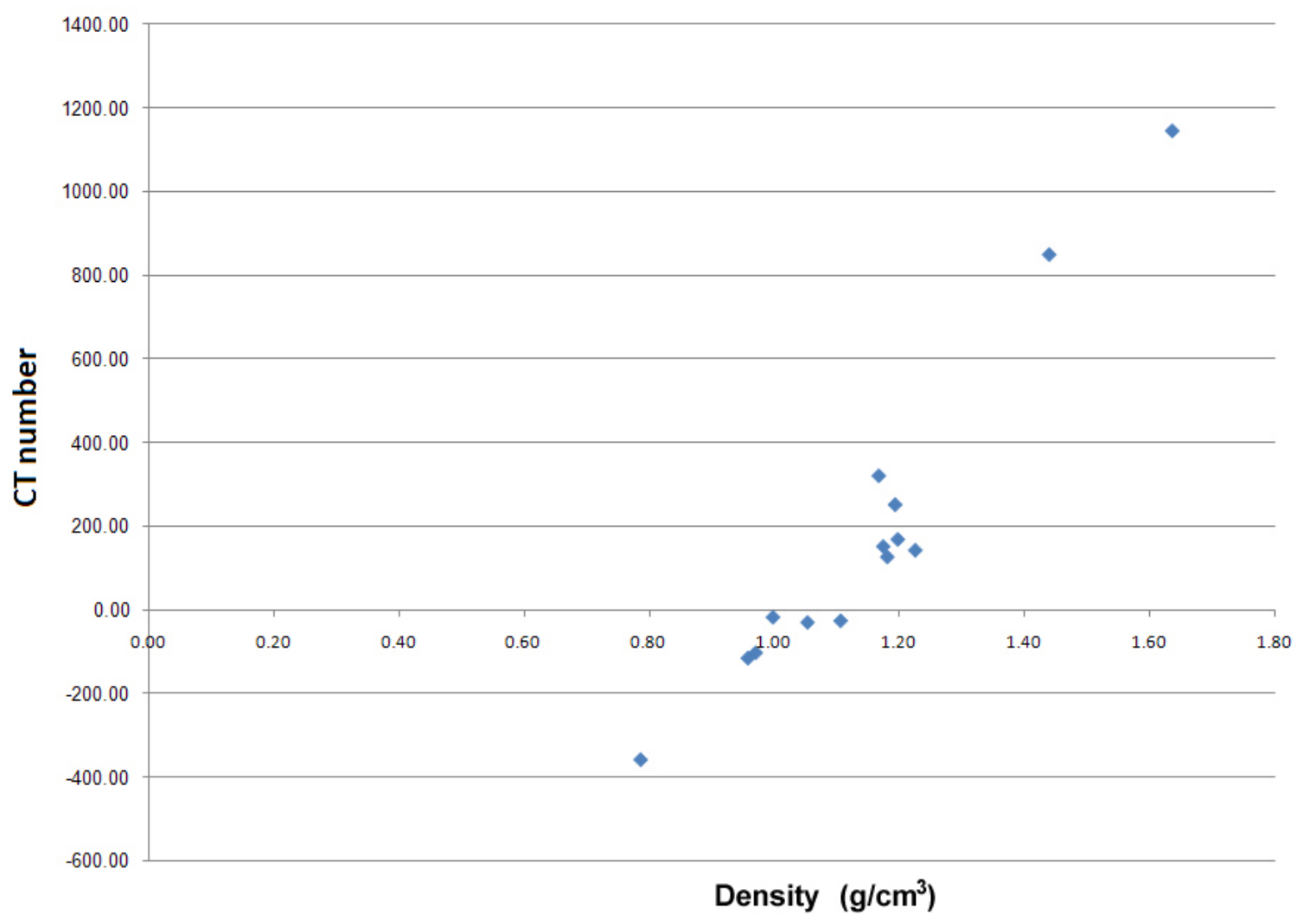

Figure 4: Relationship between CT number and average sample density

Table 3 shows the CT number mean and standard deviation (SD) for the AM samples scanned in air and in contact with the tissue equivalent head phantom (only the solid samples are included). The mean CT number ranged from a minimum -358 to a maximum of over 1200. It is interesting to note that many of the AM sample CT number ranges coincide with or are similar to those of human tissues as shown in Table 1. For example, samples 1 and 2 have CT number ranges that are similar to cortical bone, which may range from 200 to 1200 . Samples 4, 6, 7 and 8 are similar to spongious (cancellous) bone with a CT number range 50 
to 300. Sample 16 has a value similar to water when scanned in air (CT number $=0$ ) but shifted by approximately 20 CT numbers when adjacent to the phantom. Both samples 9 and 10 have mean CT numbers, which are very similar to the range found for fat tissue in the body at approximately -100 . The standard deviation of the sample CT numbers range from approximately 16 to nearly 90, the larger deviations measured in samples, which had a higher CT number. This is in keeping with CT scans of human tissue where bone (CT number > 300) has the highest standard deviation due to increased noise present in that tissue type, whilst air (CT number $=-1024)$ had the lowest standard deviation. The standard deviation of the measurements within the AM samples was due to two factors, inherent noise due to the CT imaging system and any material density variation within structure of the AM sample.

\begin{tabular}{|c|c|c|c|c|}
\hline \multirow{2}{*}{$\begin{array}{c}\text { Sample } \\
\text { number }\end{array}$} & \multicolumn{2}{|c|}{ Air } & \multicolumn{2}{c|}{ Head Phantom } \\
\cline { 2 - 5 } & Mean & SD & Mean & SD \\
\hline 1 & 977.70 & 76.02 & 850.17 & 51.28 \\
\hline 2 & 1260.98 & 89.29 & 1146.41 & 71.72 \\
\hline 3 & -11.67 & 16.31 & -17.80 & 29.88 \\
\hline 4 & 178.91 & 17.55 & 168.50 & 28.57 \\
\hline 5 & 358.96 & 20.40 & 320.82 & 27.62 \\
\hline 6 & 279.19 & 18.93 & 251.57 & 26.35 \\
\hline 7 & 148.30 & 16.30 & 142.43 & 28.67 \\
\hline 8 & 136.37 & 17.25 & 126.44 & 26.05 \\
\hline 9 & -110.38 & 21.78 & -115.74 & 34.43 \\
\hline 10 & -98.31 & 22.11 & -102.86 & 32.61 \\
\hline 12 & -358.25 & 19.64 & -358.93 & 31.08 \\
\hline 14 & 168.63 & 42.48 & 151.60 & 46.01 \\
\hline 16 & -3.49 & 24.80 & -26.37 & 29.88 \\
\hline 18 & -26.64 & 19.25 & -30.21 & 26.34 \\
\hline
\end{tabular}

Table 3: CT number average and standard deviation of AM samples scanned in air and adjacent to tissue equivalent phantom

The difference in the CT number of the samples when scanned in air compared to the CT number when attached to a soft tissue equivalent head phantom are shown in Figure 5. Note that the differences were small $(<20)$ for samples within the CT number range -360 to 320, whilst there are more significant deviations for samples with CT number 850 and 1150. In general, the CT numbers measured from the samples scanned in air were greater than that recorded from the samples scanned against the tissue phantom. 




Figure 5: The shift in CT numbers due to the presence of a tissue equivalent phantom Generally, there was no internal structural variation visible in the CT images. There were also no signs of voids or cracking in any of the samples scanned. As would be expected from an understanding of the respective RP processes, the FDM, SLA and SLS samples appeared to have uniform density throughout. This is shown using samples 3, 5, 18 shown in Figure 6 . This can also been seen in the generally flat CT number profile taken lengthwise through sample 2, shown in Figure 8. However, sample 1 shows a variation in pixel value across the sample due to the manufacturing process.

Whilst it is known that certain AM processes produce inherently porous parts, the porosity is not apparent in the CT images. This is because the porosity is at a very small scale compared to the resolution of the CT scanner and appears uniform throughout the parts. If we consider the example of SLS, the process works by sintering together thermoplastic particles with a typical average particle size of around 60 microns (material data sheet for PA2200, EOS GmbH, Munich, Germany). The particles do not fully melt but fuse together to form a sintered, porous structure. Therefore, it is reasonable to assume that this results in a slight lowering of the CT number compared to fully dense nylon produced by injection moulding, extrusion or casting. Further work will be conducted to ascertain whether the difference between SLS nylon and solid nylon can be detected in CT images. 




A

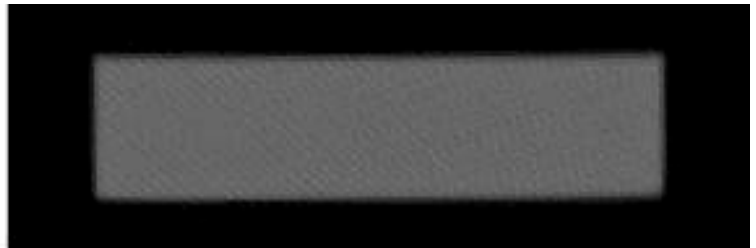

B

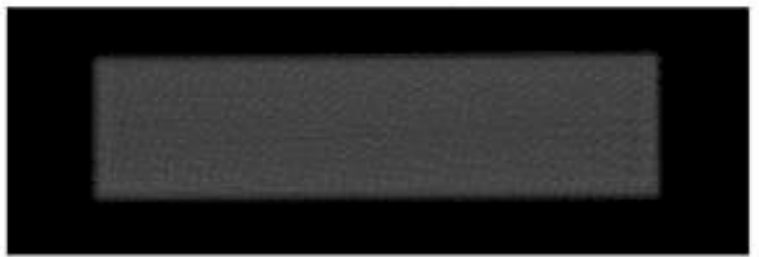

C

Figure 6: Selected sample images; $A$ = sample 3 EOS P100 Formiga; B = SLA DSM XC11122; C = FDM PC-ABS solid

However, a distinct variation of internal density was visible in the images of the 3D printed samples, 1 and 2, as shown in Figure 7. A variation in density can be seen in the CT images, which show a higher density around the periphery. This is a result of the 3D printing process, whereby the manufactured part is initially very fragile. The parts are therefore subjected to infiltration of a liquid hardener, typically a cyano-acrylate resin (as in sample 1) or wax (as in sample 2). It is known that the hardeners penetrate into the part through capillary action but that this penetration is limited to a few millimetres. This leads to a higher density "skin" or "shell" that is clearly visible in the CT image and CT number profile. The variation can also be clearly seen in the peaks in the CT number profile for sample 1, shown in Figure 8 and can be compared to the much flatter profile for sample 5 .

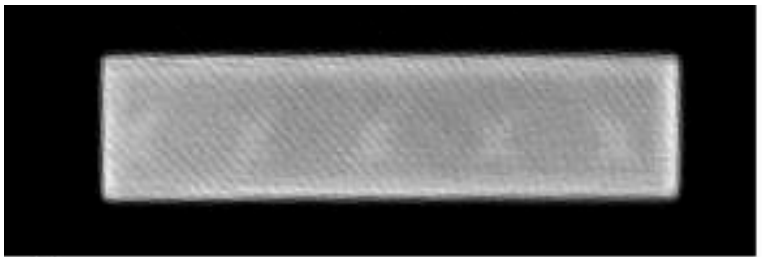

A

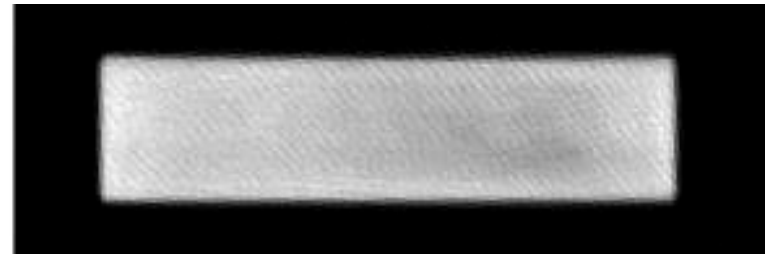

B

Figure 7: 3D Printing sample images; A = sample 1 ZCorp Z450 ZBond; B = sample 2 ZCorp Z450 ZP130 


\section{CT line profiles for Z Corp samples}



Figure 8: CT number profiles for sample 1 and 2

Each of the AM materials was inspected for the presence of beam hardening artefact. This is visible in CT scans covering areas of the body where dense material is adjacent to much less dense material. It is particularly noticeable where dense or thick bone is near soft tissue. On inspection of the CT scans beam hardening artefact was detected near the two Z Corp samples 1 and 2. These are the materials with the highest CT number and no other artefact was detected for any of the other samples. Figure 9 shows the slight but visible beam hardening effect near the $\mathrm{Z}$ Corp samples 1 and 2 as indicated by the arrows.

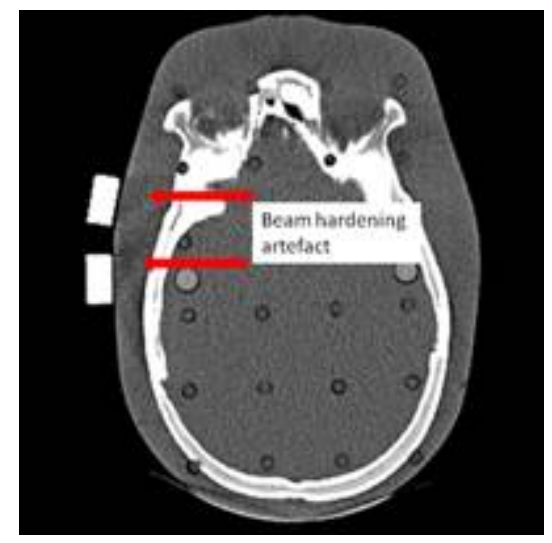

Figure 9: Beam hardening adjacent to samples 1 and 2 (image contrast enhanced)

\section{Discussion}

There are many potential applications of the information resulting from this study. Knowledge of the CT number for a particular material may enable medical devices or rehabilitation products to be designed to exhibit a specific appearance in CT images. For example, the ability to predict the appearance of a particular material in CT images could be used to either match a desired CT number in order to replicate another material or to provide 
a known image contrast with other adjacent materials. Knowledge of the CT number may simply be useful when distinguishing between a medical device and adjacent tissues in subsequent diagnoses. As the number and variety of medical applications of AM grows both inside and outside the human body it will be increasingly important to be able to predict the appearance of AM materials in CT images.

The information may also find application in the design and manufacture of immobilisation devices that can secure a patient in a given position during scanning and yet be easily distinguished from other materials or human tissues in the subsequent CT images. This would enable efficient isolation and removal an artificial object from CT images through segmentation of the object according to its CT number range. It could also enable the production of custom-fitting support devices, orthotics or prostheses that display particular characteristics in CT images. Such devices may be appropriate for patients requiring a longterm, wearable device or prosthesis who might be expected to undergo further, or repetitive radiological procedures.

These findings demonstrate that there is significant potential to use AM materials for sophisticated test objects for use in medical image modality testing. Some AM materials have CT numbers very similar to those of human tissues, as summarised in Table 4, and therefore may be used to develop anatomically accurate phantoms produced from CT scans using AM. Phantoms designed using these materials may have the added advantage of having CT numbers corresponding to real tissues. Anatomically complex, multi-tissue phantoms could be developed from existing patient CT scan data using well established image segmentation techniques providing more accurate phantoms for test purposes. The potential to expand the application to radiation dosimetry for diagnostic and therapeutic procedures is obvious.

\begin{tabular}{|c|l|l|l|}
\hline No. & \multicolumn{1}{|c|}{ AM Machine } & \multicolumn{1}{|c|}{ AM Material } & \multicolumn{1}{|c|}{ CT image equivalent tissue } \\
\hline 1 & Z Corp 450 & Z Bond (cyano-acrylate) & Cortical bone \\
\hline 2 & Z Corp 450 & ZP130 (wax) & Cortical bone \\
\hline 4 & 3D Systems 250 solid state laser & ProtoCast AF19120, DSM Somos & Spongious bone \\
\hline 6 & 3D Systems 250 solid state laser & 9420 EP (white), DSM Somos & Spongious bone \\
\hline 7 & 3D Systems 250 solid state laser & RenShape SL Y-C 9300, Huntsman & Spongious bone \\
\hline 8 & 3D Systems InVision SD & VisiJet SR & Spongious bone \\
\hline 9 & Dimension 1200 SST & ABS & Fat \\
\hline 10 & Fortus 400mc & ABS (solid) & Fat \\
\hline 16 & Fortus 400mc & PC (solid) & Water \\
\hline
\end{tabular}

Table 4: Potential AM materials that mimic human tissues in CT images

The similarity between the CT numbers for some of the samples and human tissues can also be visually illustrated by utilising preset thresholds for specific tissues available in CT image analysis software. For example, the preset threshold for bone in Mimics software (226 and higher) perfectly segments sample 1 as shown in figure 10 . Similarly, the preset thresholds for spongious bone (148 to 661) provides a very good segmentation of sample 6 and the preset thresholds for fat (-205 to -51) provides a good segmentation of sample 10. 


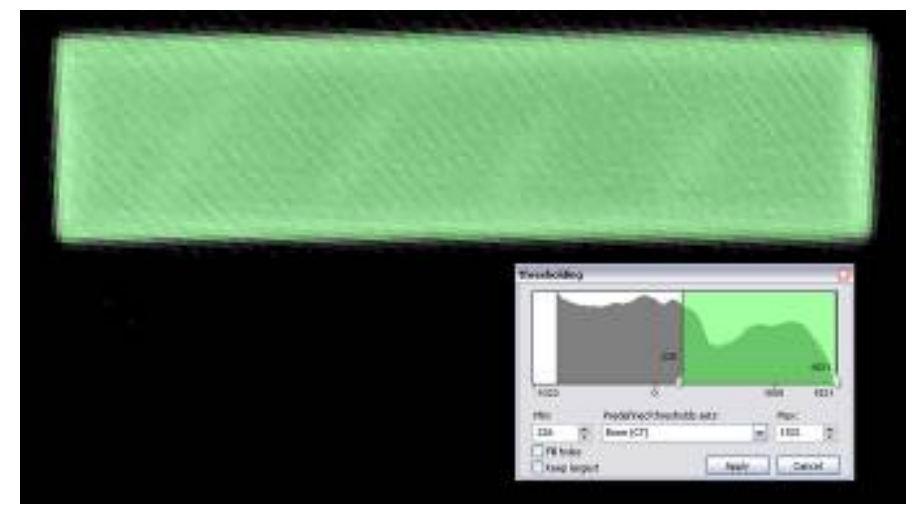

Figure 10: Effect of using preset thresholds for bone on sample 1

Figure 11 shows a CT scan of a spine model manufactured using a Z Corp 3D Printing system (the same systems as used to produce samples 1 and 2). The CT number range for the cortical bone is 1000 to 1300 whilst the range within the bone is 490 to 815 . This mimics the CT number ranges for human cortical and spongious bone closely. As described previously this is due to the AM process, which hardens the outer few millimetres of the model, resulting in an elevated CT number at the periphery. This relatively simple example demonstrates the potential to manufacture anatomically correct, sophisticated test objects with a mixture of hard and soft tissue materials, useful in radiation therapy dosimetry experiments where test objects may be created from a combined approach to model creation.

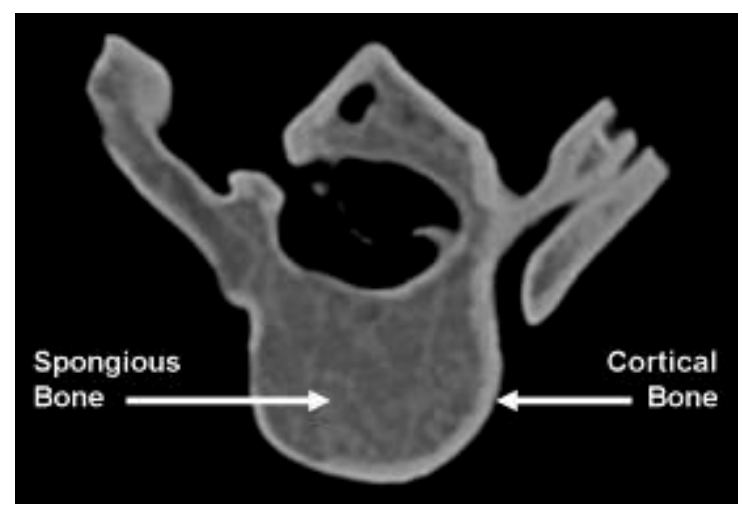

Figure 11: CT image of a 3D printed anatomical model

Anthropomorphic phantoms have been developed for use in radiation dose studies for diagnostic radiology and therapeutic radiology. Soft-tissue, lung and bone equivalent tissue substitutes (at diagnostic X-ray energy range $80-120 \mathrm{kVp}$ ) were created from urethane based compounds mixed with other materials ${ }^{21}$. This particular phantom suffered from manufacturing difficulties, in that moulds would display variation in depth or suffer from physical distortion. AM has the capability to provide accurate anatomical definition, geometrical shape and the appropriate X-ray attenuation.

\section{Future work}

The authors plan to repeat the experiments with more AM materials and develop a comprehensive database of CT numbers for a wider selection of AM materials. For the benefit of comparison, the authors also plan to include samples of well-known conventional materials such as ultra high molecular weight polyethylene and silicone. This work set out to demonstrate the appearance of AM materials in CT imaging rather than fully characterise all AM sample properties. However, further physical characterisation would complement this work and may enable relationships between physical properties and CT number to be 
ascertained. For example, nano- or micro-indentation techniques could be useful to demonstrate other properties of the samples. A porosity measure would also be useful, as this will have an effect on sample density. However, the particle size typically encountered in AM powder materials is an order of magnitude smaller than the pixel size of a typical CT image. Therefore, it is likely that CT image noise would dominate any variation in image appearance rather than porosity of the samples, which would occur at a much smaller physical scale and not show directly on the images.

As mentioned earlier, many AM processes can produce quasi-hollow structures, as shown in Figure 12. The profile for this sample can also been seen in Figure 8. These structures can be varied in section to simulate different or varying densities throughout the volume of a part. These volumes could be filled with fluids such as water, fat or oil in order to simulate different tissues or even whole organs.

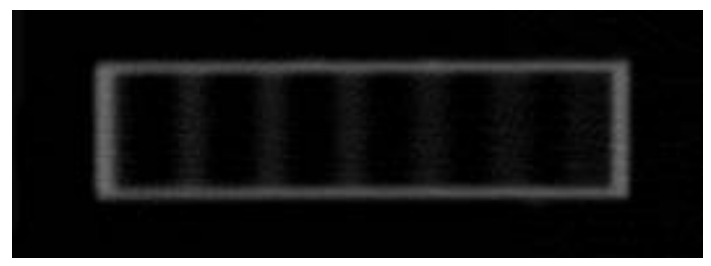

Figure 12: CT image of a quasi-hollow FDM part - sample 19

A potential advantage of AM is the ability to build objects with multiple materials and graded mixtures of materials. It is anticipated that further work in this area will prove particularly interesting when investigating objects made using AM machines that are capable of depositing multiple materials simultaneously such as the Objet Connex machines (Objet Geometries Ltd., Rehovot, Israel). This will be particularly interesting as the machines are capable of producing graded structures where material composition can be varied to produce areas of differing physical properties within a single object manufactured in a single step process. Therefore, material compositions could be adjusted to replicate a combination of specific tissues or whole sections of anatomy.

\section{Conclusions}

This study has revealed several interesting facts relating to AM materials in CT images. Firstly, the images provide an indication of material uniformity of density at the macro scale. This analysis can be used to corroborate other observations from visual analysis and mechanical testing. Secondly, the actual CT numbers of a number of commonly used AM materials has been established. This may enable the specification of AM materials for specific medical devices that are required to present a specific CT number or characteristic in CT images. Further work is required to analyse a greater variety of AM materials and in particular samples from AM processes that produce mixed, graded and multiple material parts.

\section{Acknowledgements}

The authors would like to thank the following for the donation of the samples used in this study. Dr. Russ Harris, Wolfson School of Mechanical and Manufacturing Engineering, 
Loughborough University, UK; Dr. Dominic Eggbeer, National Centre for Product Design \& Development Research, University of Wales Institute Cardiff and Jeremy Slater, Technical Sales Engineer, Design Engineering Group, Laser Lines Ltd., Banbury UK.

\section{Manufacturer Contact Details}

3D Systems Corporation, 333 Three D Systems Circle, Rock Hill, SC 29730, USA http://www.3dsystems.com

AnalyzeAVW V9.0, Lenexa, Kansas, USA

http://www.analyzedirect.com

DSM Somos, 1122 St. Charles Street, Elgin, IL 60120, USA

http://www.dsm.com/en_US/html/dsms/home_dsmsomos.htm

EOS GmbH, Robert-Stirling-Ring 1, D-82152 Krailling, Munich, Germany

http://www.eos.info/en/home.html

Huntsman Advanced Materials (UK) Ltd, Ickleton Road, Duxford, Cambridge, CB22 4XQ, UK

http://www.huntsman.com/advanced_materials

Materialise NV, Technologielaan 15, 3001 Leuven, Belgium

www.materialise.com/mimics

Objet Geometries Ltd., 2 Holtzman St., Science Park, P.O. Box 2496, Rehovot 76124, Israel

http://www.objet.com/

Stratasys, Inc., 7655 Commerce Way, Eden Prairie, MN 55344-2020, USA

http://www.dimensionprinting.com

http://www.fortus.com

Z Corporation, 32 Second Avenue, Burlington, MA 01803, USA

http://www.zcorp.com 


\section{$\underline{\text { References }}$}

1. Chua CK, Leong KF, Lim CS, Rapid Prototyping: Principles and Applications ( ${ }^{\text {rd }}$ edition), WSPC, 2010, ISBN-13: 978-9812778987

2. Gibson I, Rosen DW, Stucker B, Additive Manufacturing Technologies: Rapid Prototyping to Direct Digital Manufacturing, Springer, 2009, ISBN-13: 978-1441911193

3. Noorani RI, Rapid Prototyping: Principles and Applications, John Wiley \& Sons, 2005, ISBN-13: 978-0471730019

4. Hopkinson N, Hague R, Dickens P (eds), Rapid Manufacturing: An Industrial Revolution for a Digital Age: An Industrial Revolution for the Digital Age, Wiley Blackwell, 2005, ISBN-13: 978-0470016138

5. Arvier JF, Barker TM, Yau YY, D'Urso PS, Atkinson RL, McDermant GR, “Maxillofacial biomodelling”, Br J Oral Maxillofac Surg, 1994; 32(5): 276-83

6. Giannatsis J, Dedoussis V, "Additive fabrication technologies applied to medicine and health care: a review", International Journal of Advanced Manufacturing Technology 2009; 40(1-2): 116-127

7. Azari A, Nikzad S, "The evolution of rapid prototyping in dentistry: a review”, Rapid Prototyping Journal 2009; 15(3): 216-25

8. Bibb R, Medical modelling: the application of advanced design and development technologies in medicine, Woodhead Publishing Ltd., 2006, ISBN: 1-84569-138-5

9. Gibson I (ed), Advanced Manufacturing Technology for Medical Applications: Reverse Engineering, Software Conversion, and Rapid Prototyping, Wiley Blackwell, 2005, ISBN-13: 978-0470016886

10. Petzold R, Zeilhofer H, Kalender W, "Rapid prototyping technology in medicine-basics and applications”, Computerised Medical Imaging \& Graphics 1999; 23: 277-84

11. Webb PA, “A review of rapid prototyping (RP) techniques in the medical and biomedical sector”, J Med Eng Technol 2000; 24(4): 149-53

12. Bibb R, Eggbeer D, Evans P, "Rapid prototyping technologies in soft tissue facial prosthetics: current state of the art", Rapid Prototyping Journal 2010; 16(2): 130-7

13. Bibb R, Eggbeer D, Evans P, Bocca A, Sugar AW, "Rapid Manufacture of Custom Fitting Surgical Guides”, Rapid Prototyping Journal 2009; 15(5): 346-54

14. Bibb R, Eggbeer D, Williams R, "Rapid manufacture of removable partial denture frameworks”, Rapid Prototyping Journal 2006; 12(2): 95-9

15. Peltola SM, Melchels FP, Grijpma DW, Kellomäki M, “A review of rapid prototyping techniques for tissue engineering purposes”, Ann Med, 2008; 40(4): 268-80

16. Leong KF, Chua CK, Sudarmadji N, Yeong WY, "Engineering functionally graded tissue engineering scaffolds”, J Mech Behav Biomed Mater, 2008; 1(2): 140-52

17. Pilipović A, Raos P, Šercer M, "Experimental analysis of properties of materials for rapid prototyping”, Int J Adv Manuf Technol 2009; 40: 105-15

18. Kalender WA, "Variables and Procedures for Sequential CT", in: Computed Tomography ( $2^{\text {nd }}$ ed), Wiley VCH; 2000, pp 101, ISBN: 978-3895780813

19. Shikhaliev PM, "Beam hardening artefacts in computed tomography with photon counting, charge integrating and energy weighting detectors: a simulation study”, Phys Med Biol 2005; 50(24): 5813-27

20. Brooks RA, Di Chiro G, "Beam hardening in x-ray reconstructive tomography", Phys Med Biol 1976; 21(3): 390-8

21. Winslow JF, Hyer DE Ryan, Fisher F, Tien CJ, Hintenlang DE, "Construction of anthropomorphic phantoms for use in dosimetry studies”, J Appl Clin Med Phys 2009; 10(3): 195- 204 